\title{
Metastatic melanoma and immunotherapy-related uveitis: an incidence in Northern Ireland
}

\author{
Michael J. Dolaghan ${ }^{1} \cdot$ Bode Oladipo $^{2} \cdot$ Carole A. Cooke $^{3} \cdot$ Clara E. McAvoy $^{1}$
}

Received: 8 May 2019 / Accepted: 8 May 2019 / Published online: 21 May 2019

(c) The Royal College of Ophthalmologists 2019

To the Editor,

Inflammatory eye disease, namely uveitis, is a welldocumented adverse effect of immunotherapy, a novel treatment option that has revolutionised the outcomes for patients with metastatic melanoma. By blocking immune checkpoint proteins CTLA-4 (Ipilimumab) and PD-1 (Nivolumab, Pembrolizumab) on the surface of T-lymphocytes, the drugs cause the desired effect of tumour regression [1], but can also result in numerous immune-related adverse effects (irAEs) [2]. The incidence of uveitis secondary to CTLA-4 and PD-1 blockade in melanoma patients is said to be less than $1 \%[3,4]$. The phenomenon has also been reported in patients treated with BRAF/MEK inhibitor-targeted therapies (Dabrafenib/Trametinib/Vemurafenib) [5].

In this article, we present the incidence of uveitis in all patients in Northern Ireland treated with immunotherapy and targeted therapies for metastatic melanoma since the inception of the use of these agents in 2012. Of the 214 patients treated with immunotherapy between 2012 and 2018, six developed uveitis (five bilateral anterior and one panuveitis), a calculated incidence of $2.8 \%$, significantly higher than quoted in the literature $(<1 \%)[3,4]$. For BRAF/ MEK inhibitor-targeted therapies, of the 81 patients

I declare that the material presented is original research, has not been previously published and has not been submitted for publication elsewhere while under consideration.

Michael J. Dolaghan

mdolaghan01@qub.ac.uk

1 Department of Ophthalmology, Royal Victoria Hospital, Belfast Heath and Social Care Trust, Belfast, UK

2 Department of Medical Oncology, Northern Ireland Cancer Centre, Belfast City Hospital, Belfast Health and Social Care Trust, Belfast, UK

3 Department of Ophthalmology, Altnagelvin Area Hospital, Western Health and Social Care Trust, Londonderry, UK treated there were four cases of uveitis (two bilateral anterior, one intermediate and one panuveitis), an incidence of $4.9 \%$.

We now present two interesting illustrative cases as examples of patients with metastatic melanoma treated with immunotherapy and how their ocular immune-related adverse effects were managed.

Our first case is a 77-year-old woman with metastatic melanoma treated with Pembrolizumab therapy. During her second cycle, she developed blurred vision in her left eye, hearing loss and marked poliosis of her eyelashes. Ocular

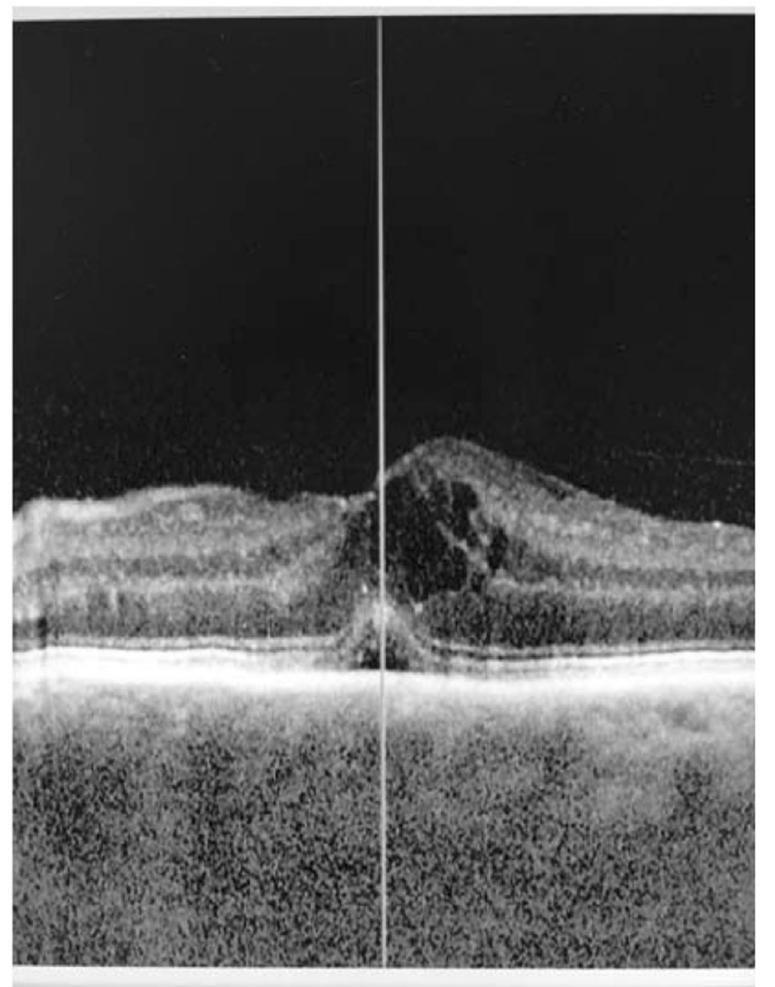

Fig. 1 OCT left eye showing resolving intra-retinal fluid with oral steroids 
1 - Scotopic 0.01 ERG

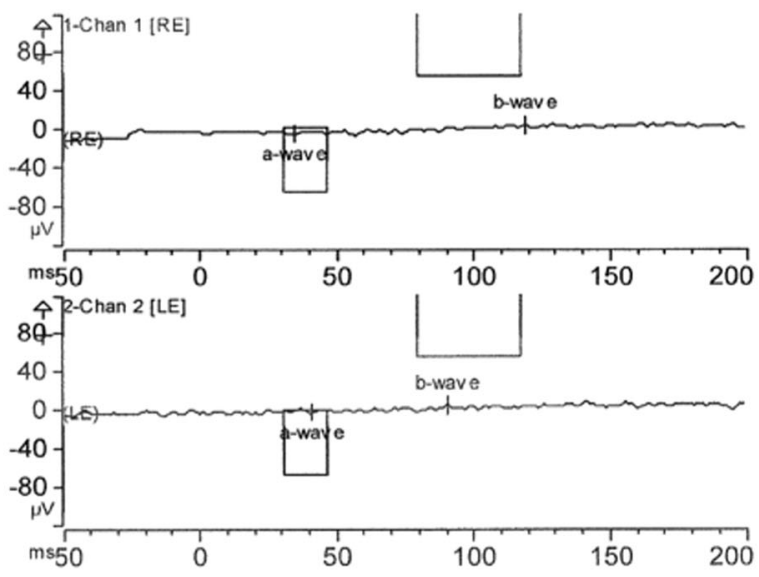

3 - Scotopic 20.0 ERG
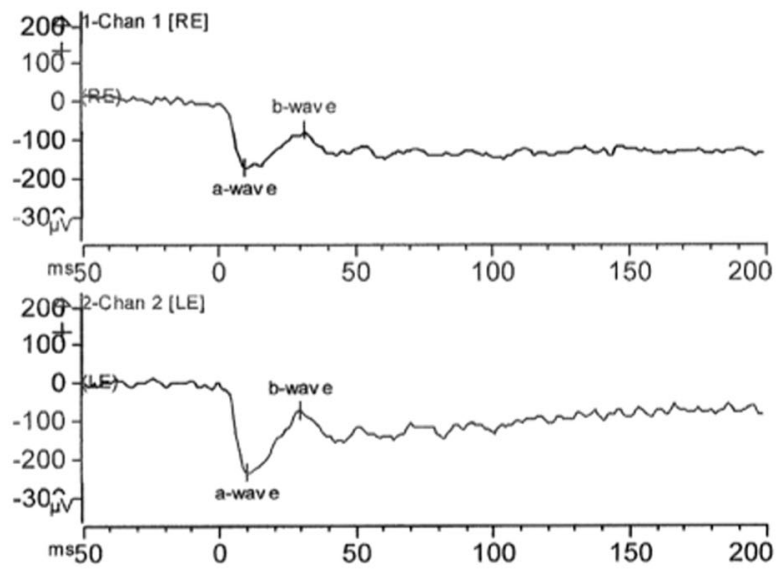

5 - Photopic 3.0 ERG
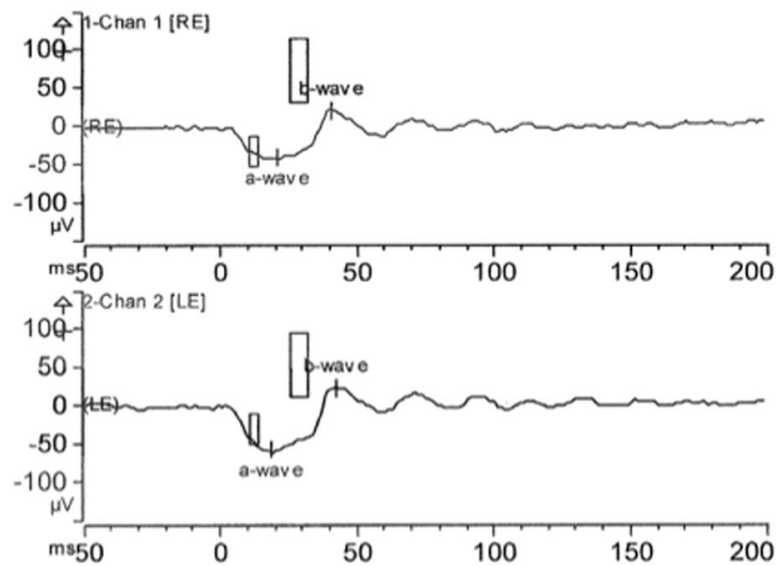

2 - Scotopic 3.0 ERG
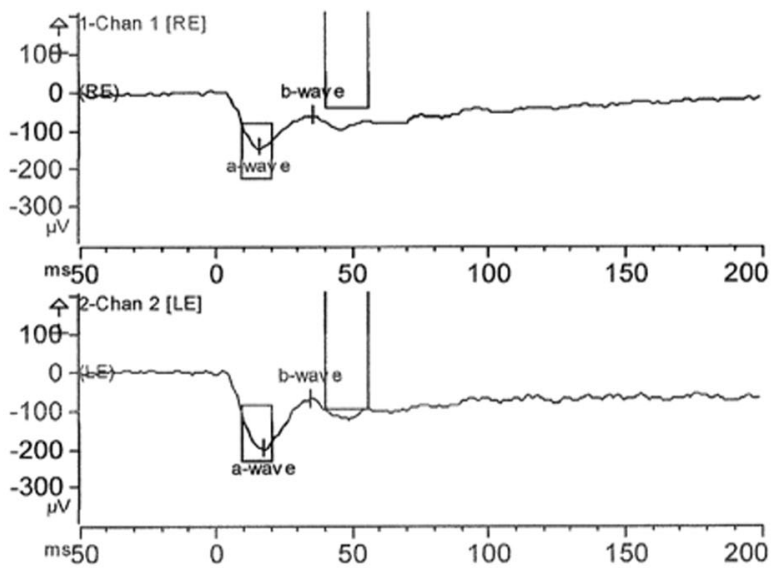

4 - scotopic 3.0 OP's
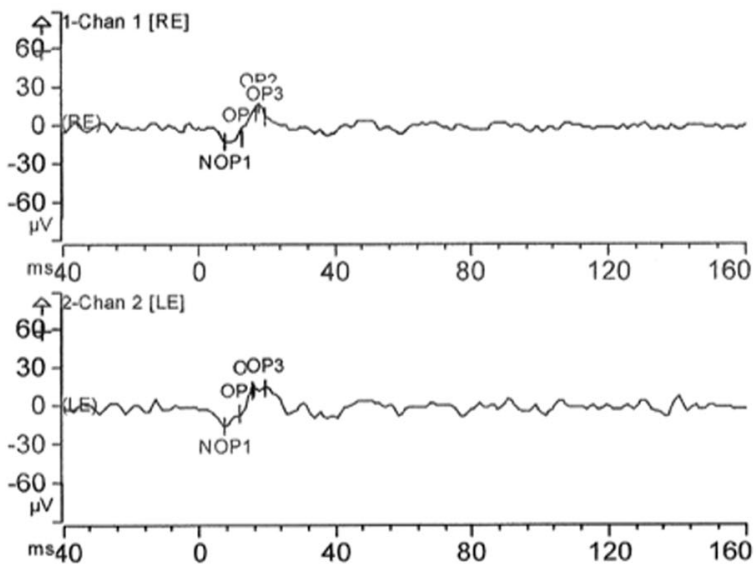

\section{6 - Photopic 3.0 flicker}
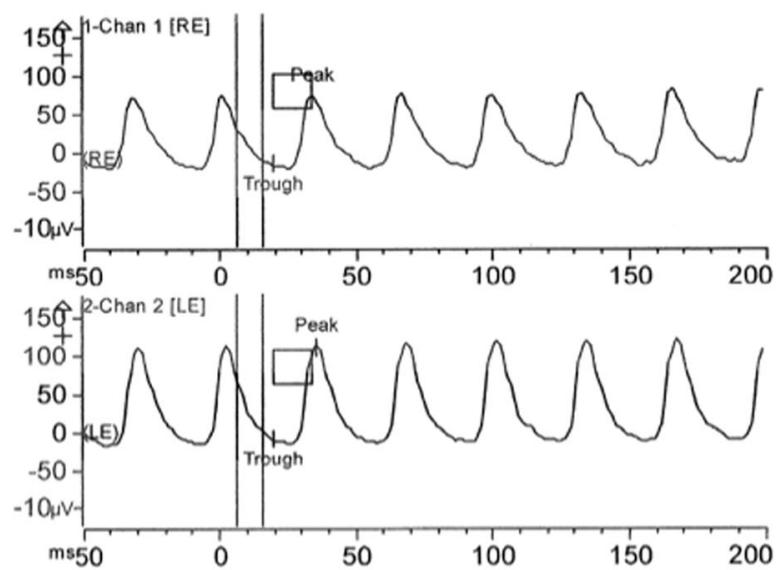

Fig. 2 Electroretinography (ERG) suggestive of melanoma-associated retinopathy (MAR)—absent rod function in scotopic ERG, and selective reduction of the 'b-wave' with preservation of the 'a-wave' resulting in a negative type ERG overall

examination revealed bilateral panuveitis with exudative retinal detachments, which was worse in the left eye. It was felt that this patient had developed a VKH (Vogt Koyanagi Harada)-like syndrome secondary to her immunotherapy.
Her optical coherence tomography and vision improved with oral steroids and topical PredForte (Fig. 1).

A 72-year-old gentleman with metastatic melanoma is the focus of our second case. Treated with two cycles 
of Ipilimumab/Nivolumab and five cycles of Pembrolizumab, he developed bilateral anterior uveitis and raised intra-ocular pressures (IOPs). His ocular inflammation was treated with Maxidex, and IOPs controlled with Latanoprost and Betagan. Unfortunately he developed further irAEs, including grade 2 colitis, adrenal insufficiency and diabetes. His immunotherapy was discontinued due to cumulative toxicity despite complete radiological response. Interestingly, after the resolution of all ocular inflammation, poor vision in the left eye persisted (6/24) and electrophysiology led to a diagnosis of melanoma-associated retinopathy (MAR), a paraneoplastic autoimmune complication of melanoma (Fig. 2). This was confirmed by the presence of anti-recoverin and carbonic anhydrase II antibodies.

Clinicians must be vigilant for inflammatory adverse effects of immunotherapy in the eye. Often the effects are minor; however, rarely, certain cases can be sight threatening. None of our patients had immunotherapy discontinued due to ocular irAEs alone, and all were managed with topical and oral steroids. Our data highlight the higher-thananticipated incidence of ocular inflammation in patients on novel therapies for metastatic melanoma in Northern Ireland.

\section{Compliance with ethical standards}

Conflict of interest The authors declare that they have no conflict of interest.

Publisher's note: Springer Nature remains neutral with regard to jurisdictional claims in published maps and institutional affiliations.

\section{References}

1. Karimkhani C, Reddy BY, Dellavalle RP, et al. Novel therapies for unresectable and metastatic melanoma. BMJ. 2017;359:j5174.

2. Andrews S, Holden R. Characteristics and management of immune related adverse effects associated with Ipilimumab, a new immunotherapy for metastatic melanoma. Cancer Manag Res. 2012;4:299-307.

3. Hahn L, Pepple KL. Bilateral neuroretinitis and anterior uveitis following Ipilimumab treatment for metastatic melanoma. J Ophthalmic Inflamm Infect. 2016;6:14.

4. Hanna KS. A rare case of pembrolizumab-induced uveitis in a patient with metastatic melanoma. Pharmacotherapy. 2016;36:e183-8.

5. Sarny S, Neumayer M, Kofler J, El-Shabrawi Y. Ocular toxicity due to Trametinib and Dabrafenib. BMC Ophthalmol. 2017;17:146.

\title{
Self-tattooing of eyeball with inadvertent corneoscleral perforation: the implication of social media
}

\author{
Jia Yu Ng${ }^{1} \cdot$ Darren Shu Jeng Ting $\mathbb{D}^{1,2} \cdot$ Daniela Vaideanu-Collins ${ }^{1} \cdot$ Hardeep Singh Mudhar ${ }^{3} \cdot$ Bart Wagner $^{4} \cdot$ \\ Patricia Goggin $^{5}$. Andrew Gibson ${ }^{1} \cdot$ Trushar Patel $^{1}$
}

Received: 30 April 2019 / Accepted: 2 May 2019 / Published online: 5 June 2019

(c) The Royal College of Ophthalmologists 2019

These authors contributed equally: Jia Yu Ng, Darren Shu Jeng Ting

Darren Shu Jeng Ting

ting.darren@gmail.com

1 Department of Ophthalmology, The James Cook University Hospital, Middlesbrough, UK

2 Academic Ophthalmology, Division of Clinical Neuroscience, School of Medicine, University of Nottingham, Nottingham, UK

3 National Specialist Ophthalmic Pathology Service, Department of Histopathology, Royal Hallamshire Hospital, Sheffield, UK

4 Electron Microscopy Unit, Department of Histopathology, Royal Hallamshire Hospital, Sheffield, UK

5 Biomedical Imaging Unit, University Hospital Southampton NHS Trust, Southampton, UK
To the Editor

Body modification procedures are becoming increasingly popular, especially, in the social media generation where an easily accessible self-care or Do-It-Yourself (DIY) culture is common [1]. We report a case of inadvertent corneoscleral perforation following self-attempt of bilateral ocular tattooing guided by a YouTube video.

A 34-year-old Caucasian man presented to our eye casualty with an acute painful right eye after performing a DIY ocular tattooing procedure using a 31-gauge hypodermic needle, and Fibracolor white baby finger paint purchased online. After multiple prompting during the initial consultation, the patient disclosed a past history of bilateral laser epithelial keratomileusis (LASEK) for myopia, radical bilateral bulbar conjunctivalectomy for post-LASEK chronic bulbar conjunctival 